\title{
Incidental Diagnosis of a Brown Tumor Mimicking Bone and Lung Metastasis during a Parathyroid Scintigraphy
}

\author{
Fabrice Fokoué*, Sanae El Mselmi, Nadia Abaouz, Nadia Ismaili Alaoui \\ Nuclear Medicine Department, Oncology Hospital, Teaching Hospital Hassan II, Faculty of Medicine and Pharmacy, \\ Sidi Mohamed Ben Abdellah University, Fez, Morocco \\ Email: ^fabricefokoue2017@gmail.com
}

How to cite this paper: Fokoué, F., El Mselmi, S., Abaouz, N. and Alaoui, N.I. (2020) Incidental Diagnosis of a Brown Tumor Mimicking Bone and Lung Metastasis during a Parathyroid Scintigraphy. Advances in Molecular Imaging, 10, 7-13.

https://doi.org/10.4236/ami.2020.102002

Received: March 11, 2020

Accepted: April 19, 2020

Published: April 22, 2020

Copyright $\odot 2020$ by author(s) and Scientific Research Publishing Inc. This work is licensed under the Creative Commons Attribution International License (CC BY 4.0).

http://creativecommons.org/licenses/by/4.0/

\begin{abstract}
We report herein a case of a 40-year-old male patient with chronic renal failure presenting a severe hyperparathyroidism with an elevation of parathormone level evaluated in nuclear medicine department for MIBI-Technetium$99 \mathrm{~m}$ parathyroid scintigraphy. The parathyroid scintigraphy revealed the appearance of a preferential fixation of the MIBI-99mTc opposite the lower left pole of the thyroid and opposite the upper part of the right hemi thorax. A subsequent single-photon emission computed tomography-computed tomography focused on the cervico-thoracic region was performed and showed an ectopic parathyroid adenoma associated with an incidental brown tumor mimicking bone and lung metastases. Our case report confirms the usefulness of additional hybrid SPECT-CT imaging in the management of hyperparathyroidism.
\end{abstract}

\section{Keywords}

Hyperparathyroidism, Thyroid Scintigraphy, Parathyroid Scintigraphy, Technetium-99m, MIBI-Technetium-99m, SPECT/CT, Ectopic

Parathyroid Adenoma, Brown Tumor

\section{Introduction}

Brown tumors are rare osteolytic bone lesions found in $4.5 \%$ of patients with primary hyperparathyroidism (PHPT) and $1.5 \%$ to $1.7 \%$ of patients with secondary hyperparathyroidism (SHPT). They can affect the entire skeleton and the most common locations are the pelvis, ribs, mandible and hands. Localizations on the long bones are extremely rare. Brown tumors are involved in osteolytic 
bone lesions that can mimic malignant bone tumors [1]. We report herein the case of a patient referred for isotopic exploration looking for a parathyroid adenoma in a context of secondary hyperparathyroidism in which the parathyroid scintigraphy and the complement by SPECT/CT scan allowed to highlight an associated ectopic parathyroid adenoma with a brown tumor mimicking bone and lung metastases thus allowing to plane best care for this patient after an accurate diagnosis.

\section{Patient and Method}

This is a 40-year-old male patient with a story of a chronic smoking cessation and chronic hemodialysis for 5 years, referred to the Nuclear Medicine department for an isotopic exploration of a secondary hyperparathyroidism (SHPT) with a biological assessment revealing a normal calcemia at $2.3 \mathrm{mmol} / \mathrm{L}$ (Normal value: $2.2-2.6 \mathrm{mmol} / \mathrm{L}$ ) and a parathormone level raised to $3226.6 \mathrm{pg} / \mathrm{mL}$ (Normal value: 15 - 88) in favor of secondary hyperparathyroidism. The parathyroid scintigraphy was carried out in two stages according to a hybrid wash-out protocol (double tracer) with acquisition of images on a hybrid gamma camera dual head SPECT/CT Siemens Symbia T6 2010. The first step consisted in a thyroid scintigraphy with the creation of a planar recording in previous incidence $10 \mathrm{~min}$ after injection of $50 \mathrm{MBq}$ of Pertechnetate. Then the second stage consisted in the realization of a parathyroid scintigraphy by a planar recording in anterior incidence $10 \mathrm{~min}$ then 2 hours and 4 hours after injection of $550 \mathrm{MBq}$ of MIBI-99mTc followed by a tomoscintigraphy coupled with a single-photon emission computed tomography-computed tomography (SPECT/CT) scan examination [2] [3].

\section{Results}

The $99 \mathrm{mTc}$ thyroid scintigraphy made it possible to visualize a thyroid gland in a normal anatomical position and suitable fixation. While the MIBI-99mTc parathyroid scintigraphy made it possible to highlight on the early image and the late images compared to the thyroid image with $99 \mathrm{mTc}$ the appearance of a preferential fixation of the MIBI-99mTc opposite the lower left pole of the thyroid and opposite the upper part of the right hemi thorax (Figure 1) better visible on the subtraction image (Figure 2). Parathyroid Scintigraphy with MIBI-99mTc combined with the SPECT/CT scan performed 4 hours later after a cervico-mediastinal centering revealed an ectopic parathyroid adenoma behind the left sterno-clavicular joint. (Figure 3) and an osteolytic bone lesion of the 4th right rib blowing the cortex, expansive and extended to the adjacent pulmonary field (Figure 4) suggesting a brown tumor in this context.

\section{Discussion}

SHPT is the most common cause of benign hypercalcaemia and is linked in $85 \%$ of cases to a parathyroid adenoma. The main pathogenic mechanism leading to 


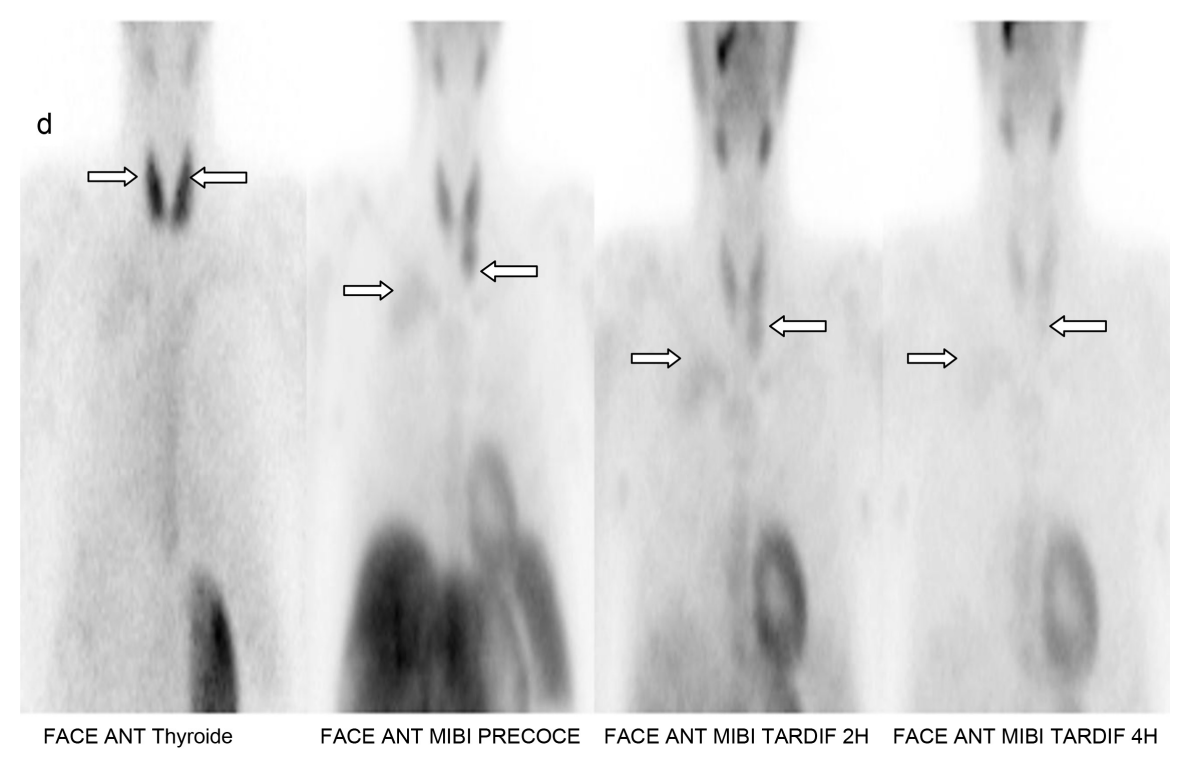

Figure 1. Thyroid Scintigraphy with $99 \mathrm{mTc}$ then with MIBI-99mTc in anterior cervico-thoracic incidence performed $10 \mathrm{~min}, 2$ hours and 4 hours after an IV injection of 550 $\mathrm{MBq}$ of MIBI-99mTc.
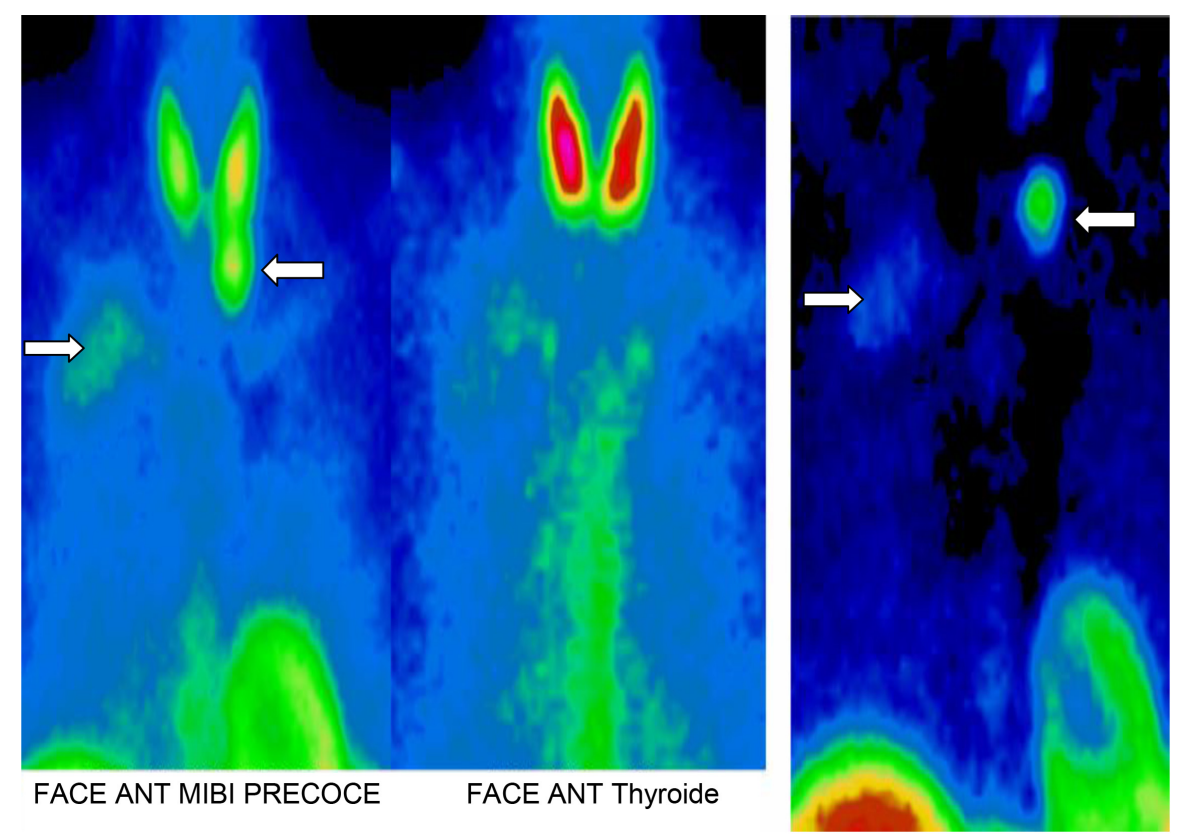

Subtraction image

Figure 2. Subtraction image showing a focal point of preferential accumulation of MIBI-99mTc projecting opposite the lower left pole of the thyroid and a second focal point facing the upper part of the right chest.

SHPT is a deficiency of 1,25-dihydroxycholecaciferol, which results in hypocalcaemia and hyperphosphatemia, leading to an increase in the production and secretion of PTH by the parathyroid gland. In 1934, Albright made the first description at the level of the facial skeleton of a Brown tumor. Brown tumors are an extreme form of manifestation of fibrocystic osteitis. The tumor lesion 


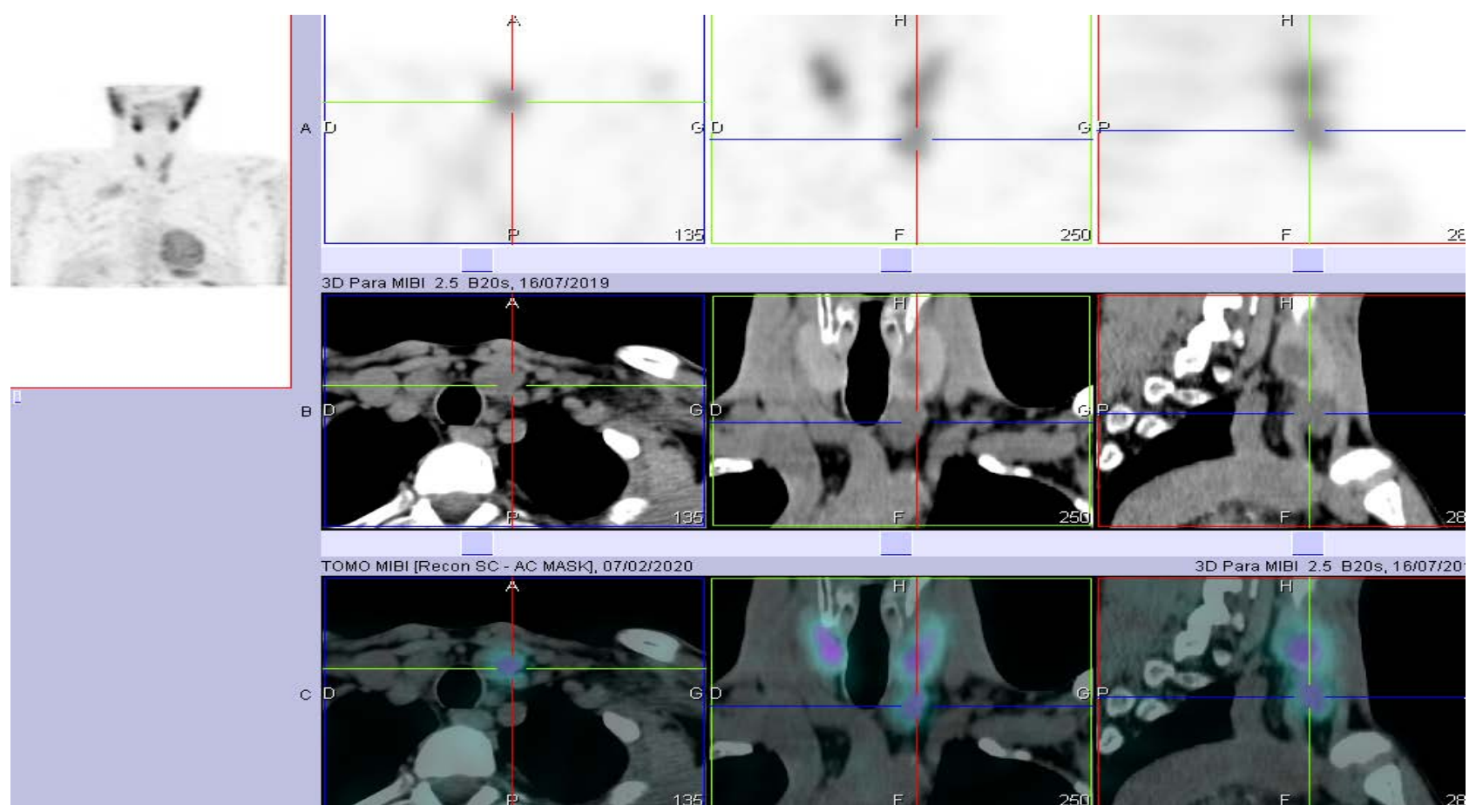

Figure 3. SPECT/CT showing an ectopic parathyroid adenoma behind the left sterno clavicular joint.

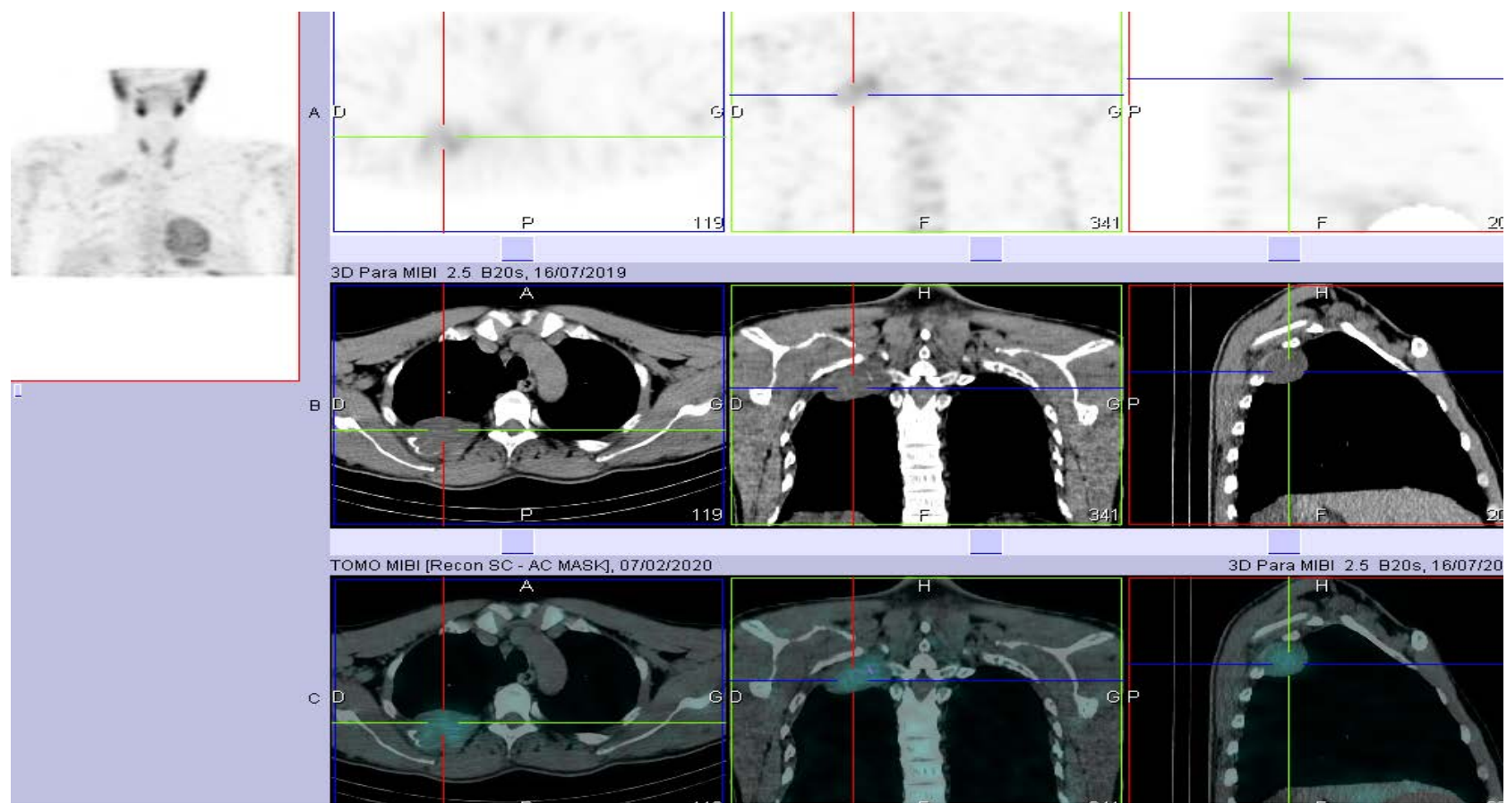

Figure 4. SPECT/CT showing an osteolytic bone lesion of the 4th right rib blowing the cortex, expansive and extended to the adjacent pulmonary field.

corresponds to trabecular resorption phenomena complicated by micro fractures with intra-lacunar bleeding. These are very vascular lesions which may contain necrotic centers and hemosiderin deposits, hence the characteristic brown color [4]. The reported prevalence of brown tumors has decreased to $<0.1 \%$. Due 
to the quality of medical care and screening in developed countries, it is increasingly rare to find an associated bone disease in secondary hyperparathyroidism. There have been reports of extensive multiple brown tumors, some of which mimic cancer metastases, due to hyperparathyroidism due to a parathyroid adenoma [5] [6] [7]. According to a review of the recent literature there are only a few reported cases of brown tumors caused by parathyroid carcinoma [5] - two cases of mandibular brown tumors and two cases of multiple brown tumors in the lower limbs [7] [9]. Symptoms related to brown tumors depend on their size, location and the nature of the adjacent structures. Bone pain, fractures and neurological deficit have been described [8] [9]. These lesions and associated symptoms usually regress after correction of hyperparathyroidism.

Parathyroid Scintigraphy is a localization examination. It should not be used to make the positive diagnosis of hyperparathyroidism, which is essentially biological. Its level of indication is variable depending on the clinical situation. MIBI labeled with $99 \mathrm{mTc}$ is a cationic and lipophilic molecule which once captured, concentrates in the mitochondria of thyroid and parathyroid cells. As the parathyroid adenomatous cells are particularly rich in mitochondria, they will capture radio pharmaceutical sequestration longer than in the thyroid [2], the Parathyroid Scintigraphy with $99 \mathrm{~m}$ Tc-MIBI therefore has good sensitivity for the detection of parathyroid adenomas. A positive scan is correlated with the size of the adenoma and the levels of ionized calcium. The procedure may include a hybrid SPECT/CT scan acquisition, which is useful for locating the pathological parathyroid gland and identifying ectopic adenomas [2]. This precise location is useful for performing a minimally invasive para thyroidectomy.

The absorption of MIBI in brown tumors and bone metastases has also been described [5]. In our case, while the MIBI-99m Tc Scintigraphy allowed us to visualize the abnormal cervical and thoracic absorption (Figure 1 and Figure 2), SPECT/CT scan allowed us to localize a lower left parathyroid adenoma (Figure 3 ) and the osteolytic bone lesion of the 4 th right rib extended to the right pulmonary field (Figure 4) evoking a brown tumor.

Multiple brown tumors without focal absorption of MIBI-99m Tc have also been described and are thought to be linked to a lack of mitochondria. In such cases, 18 F-FDG PET/CT scan could allow the detection of these tumors [10].

The goals of preventing and treating brown tumors include the normalization of calcaemia and phosphoremia. A total or a subtotal parathyroidectomy is usually done to decrease serum PTH levels. Finally a lumpectomy could be performed in certain indications [6] [9].

In our case, the diagnosis of parathyroid adenoma and Brown's tumor were confirmed by a histological examination and the parathormone level decreased considerably to a suitable value after a surgery following a multidisciplinary management.

\section{Conclusion}

Apart from the rarity and originality of the reported clinical case, our study 
highlights the key role of isotopic imaging procedures, in particular parathyroid Scintigraphy and SPECT/CT scan coupling in the exploration of hyperparathyroidism, by locating the ectopic parathyroid adenoma, diagnosing the brown tumor and defining its anatomical relationships, thus improving the management of hyperparathyroidism and allowing the medical team to anticipate an unusual situation.

\section{Conflicts of Interest}

The authors declare that they have no conflicts of interest regarding the production of this article.

\section{Ethical Approval}

Obtained by the ethics committee of the Faculty of Medicine and Pharmacy of Sidi Mohamed Ben Abdellah University of Fez.

\section{Consent to Publication}

Obtained from the patient.

\section{Availability of Data and Material}

Data sharing does not apply to this article because no data set was generated or analyzed during the current study.

\section{References}

[1] Lapras, V., Giammarile, F., Lifante, J.-C. and Peix, J.-L. (2014) Imagerie normale et pathologique des glandes parathyroïdes. EMC-Radiologie et Imagerie Médicale Cardiovasculaire Thoracique Cervicale, 9, 1-22. https://doi.org/10.1016/S1879-8535(09)72781-3

[2] Hindié, E., Billotey, C., Taïeb, D., Calzada, M., et al. (2011) Guide pour la rédaction de protocoles pour la scintigraphie des glandes parathyroïdes. Médecine Nucléaire, 35, 665-675. https://doi.org/10.1016/j.mednuc.2011.10.005

[3] Palestro, C.J., Tomas, M.B. and Tronco, G.G. (2005) Radionuclide Imaging of the Parathyroid Glands. Seminars in Nuclear Medicine, 35, 266-276. https://doi.org/10.1053/j.semnuclmed.2005.06.001

[4] Charfi, H., Nouira, M., Ezairi, F., Sfar, R., Guezguez, M. and Essabah, H. (2015) Brown Tumors Mimicking Bone and Lung Metastases: Key Role of Radionuclide Imaging. La Presse Médicale, 44, 860-864. https://doi.org/10.1016/j.lpm.2015.04.021

[5] Nilsson, I.L, Zedenius, J., Yin, L. and Ekbom, A. (2007) The Association between Primary Hyperparathyroidism and Malignancy: Nationwide Cohort Analysis on Cancer Incidence after Parathyroidectomy. Endocrine-Related Cancer, 14, 135-140. https://doi.org/10.1677/erc.1.01261

[6] Hoshi, M., Takami, M., Kajikawa, M., Teramura, K., Okamoto, T., Yanagida, I., et al. (2008) A Case of Multiple Skeletal Lesions of Brown Tumors, Mimicking Carcinoma Metastases. Archives of Orthopaedic and Trauma Surgery, 128, 149-154. https://doi.org/10.1007/s00402-007-0312-0

[7] Ben Dhaou, B., Derbali, F., Aydi, Z., Baili, L., Boussema, F. and Rokbani, L. (2013) 
Tumeurs brunes multiples révélant une hyperparathyroïdie primaire. Med Nucl, 37, 52-55. https://doi.org/10.1016/j.mednuc.2012.11.005

[8] Alfawareh, M.D., Halawani, M.M., Attia, W.I. and Almusrea, K.N. (2015) Brown Tumor of the Cervical Spines: A Case Report with Literature Review. Asian Spine Journal, 9, 110-120. https://doi.org/10.4184/asj.2015.9.1.110

[9] Radulescu, D., Chis, B., Donca, V. and Munteanu, V. (2014) Brown Tumors of the Femur and Pelvis Secondary to a Parathyroid Carcinoma: Report of One Case. Revista Medica de Chile, 142, 919-923.

https://doi.org/10.4067/S0034-98872014000700014

[10] Herrmann, K., Takei, T., Kanegae, K., et al. (2009) Clinical Value and Limitations of [11C]-Methionine PET for Detection and Localization of Suspected Parathyroid Adenomas. Molecular Imaging and Biology, 11, 356-363.

https://doi.org/10.1007/s11307-009-0205-4 